\title{
III-V/SOI vertical cavity laser with in-plane output into a Si waveguide
}

Park, Gyeong Cheol; Xue, Weiqi; Semenova, Elizaveta; Yvind, Kresten; Mørk, Jesper; Chung, II-Sug

Published in:

Proceedings of the Optical Fiber Communications Conference and Exhibition 2015

Link to article, DOI:

10.1364/OFC.2015.W2A.17

Publication date:

2015

Document Version

Peer reviewed version

Link back to DTU Orbit

Citation (APA):

Park, G. C., Xue, W., Semenova, E., Yvind, K., Mørk, J., \& Chung, I-S. (2015). III-V/SOI vertical cavity laser with in-plane output into a Si waveguide. In Proceedings of the Optical Fiber Communications Conference and Exhibition 2015 [W2A.17] IEEE. https://doi.org/10.1364/OFC.2015.W2A.17

\section{General rights}

Copyright and moral rights for the publications made accessible in the public portal are retained by the authors and/or other copyright owners and it is a condition of accessing publications that users recognise and abide by the legal requirements associated with these rights.

- Users may download and print one copy of any publication from the public portal for the purpose of private study or research.

- You may not further distribute the material or use it for any profit-making activity or commercial gain

- You may freely distribute the URL identifying the publication in the public portal 


\title{
III-V/SOI Vertical Cavity Laser with In-plane Output into a Si Waveguide
}

\author{
Gyeong Cheol Park, Weiqi Xue, Elizaveta Semenova, Kresten Yvind, Jesper Mørk, and \\ Il-Sug Chung* \\ Department of Photonics Engineering (DTU Fotonik), Technical University of Denmark, DK-2800 Kgs. Lyngby, \\ Denmark \\ *ilch@fotonik.dtu.dk
}

\begin{abstract}
We experimentally demonstrate an optically-pumped III-V-on-SOI hybrid vertical-cavity laser that outputs light into an in-plane $\mathrm{Si}$ waveguide, using CMOS-compatible processes. The laser operates $\sim 1.49 \mu \mathrm{m}$ with a side-mode suppression ratio of $\sim 27 \mathrm{~dB}$ and has a similar threshold as long-wavelength VCSELs.
\end{abstract}

OCIS codes: (130.3120) Integrated optics devices; (140.2020) Diode lasers; (200.4650) Optical Interconnects.

\section{Introduction}

The vertical-cavity surface-emitting laser (VCSEL) is widely used for short-distance optical interconnects, e.g., communications inside a large data center, due to its small energy consumption, high speed, and efficient light coupling to an optical fiber. Its energy consumption in generating an optical bit (energy/bit) can be as low as $69 \mathrm{fJ} / \mathrm{bit}$ [1]. Despite of these attractive laser characteristics, using the VCSEL for chip-level optical interconnects is not feasible since coupling output light from the VCSEL into an in-plane $\mathrm{Si}$ waveguide is not straightforward, requiring extra efforts such as a grating coupler and an alignment. Recently, we have proposed the hybrid vertical cavity laser (hVCL) structure that is a vertical cavity laser monolithically integrated onto a Si-on-insulator (SOI) wafer and can output milli-Watt-level light directly into a Si waveguide [2]. It can maintain all desirable properties of the VCSEL and its speed may exceed that of the VCSEL since it has a smaller modal volume than the VCSEL. Thus, the hVCL appears to be an attractive low-cost high-performance light source for the chip-level optical interconnects where an energy/bit less than $10 \mathrm{fJ} / \mathrm{bit}$ is not demanded. In this paper, we report the demonstration of an optically-pumped hVCL with in-plane output, which we believe is the first demonstration of lasing with in-plane emission in this structure to our knowledge.

(a)

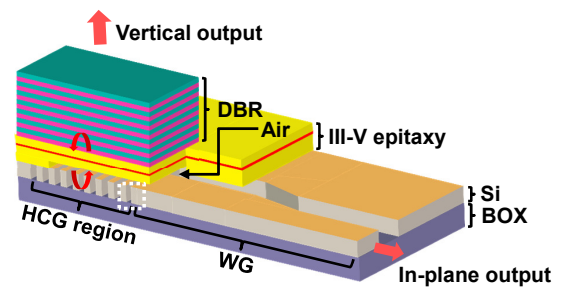

(b)

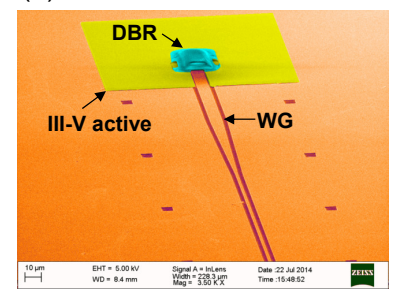

Fig. 1. (a) Schematic of the hVCL. (b) Scanning electron microscope (SEM) image of the fabricated hVCL.

\section{Device design, Fabrication, and Characterization}

As shown in Fig. 1(a), the hVCL consists of a dielectric distributed Bragg reflector (DBR), a III-V active material, and a high-index-contrast grating (HCG) region formed in the Si layer of a SOI wafer. A Si waveguide is deliberately positioned in front of the HCG region [2]. The HCG region reflects $99.5 \%$ of vertically-incident light as well as routing $0.3 \%$ of it laterally into the $\mathrm{Si}$ waveguide. Thus, light is amplified vertically between the DBR and the HCG and is emitted into the Si waveguide. This routing mostly occurs around the white dotted square in Fig. 1(a) and the routed power can be a few $\mathrm{mW}$ in the Si waveguide [2]. 
The hVCL samples were fabricated by using CMOS-compatible processes. The HCG region and waveguide were made by using e-beam lithography and dry etching. Then, the III-V active material including seven InGaAlAs/InGaAlAs quantum wells (QWs) is directly wafer bonded to the SOI wafer at $300^{\circ} \mathrm{C}$. After removing the InP substrate, 7 pairs of $\mathrm{TiO}_{2} / \mathrm{SiO}_{2}$ DBR layers are deposited. The DBR region was defined by lift-off process. Finally, to form an air gap between the III-V active material and the SOI wafer, wet sacrificial etching was conducted. The fabricated hVCL chip was cleaved in the middle of the Si waveguide to measure light output from the waveguide. A fabricated hVCL sample is shown in Fig. 1(b).

For optical pumping, a $980 \mathrm{~nm}$ pulsed laser diode with a $4 \mathrm{~nm}$ pulse width and $5 \mathrm{MHz}$ repetition rate, and alignment optics including a $50 \times$ near-infrared objective lens are used to focus a pumping beam onto the DBR. The pumping spot has a $e^{-2}$ diameter of $6 \mu \mathrm{m}$. The in-plane emission power from the $\mathrm{Si}$ waveguide was measured by using a multimode fiber, and the beam profile of the in-plane emission was scanned by using a lensed fiber, as shown in the inset of Fig. 2(b).

\section{Low threshold and Single mode property}
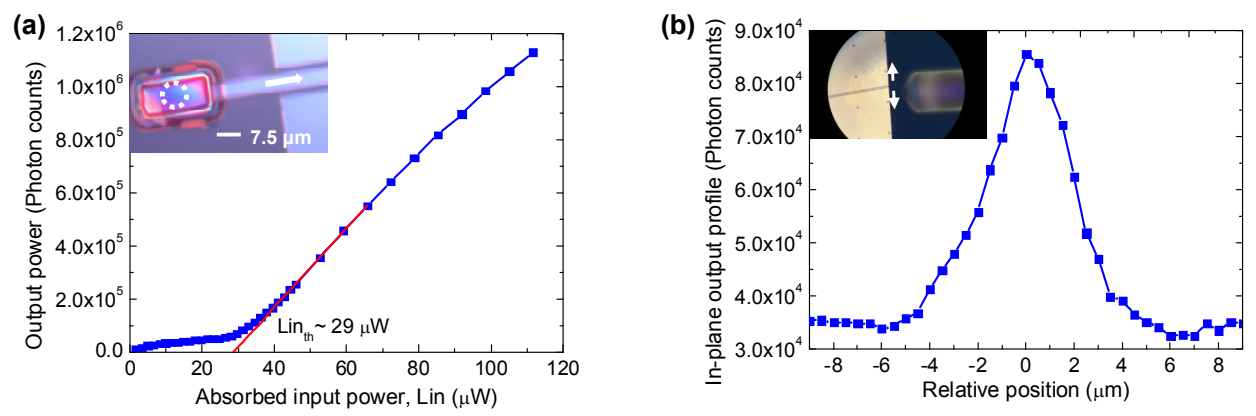

Fig. 2. (a) Measured in-plane output power as a function of absorbed pump power. (b) Beam profile of the in-plane emission scanned by using a lensed fiber.

Figure 2(a) shows the measured in-plane output power as a function of absorbed power in the QW and separate confinement heterostructure ( $\mathrm{SCH}$ ) layers. The absorbed power is estimated by using the monitored pump power, the transmittance of the alignment optics, and the absorption efficiency in the QW and SCH layers. The threshold, $P_{\text {th }}$ is $\sim 29 \mu \mathrm{W}$. The corresponding threshold current, $I_{\text {th }}$ for the continuous wave $(\mathrm{CW})$ case can be estimated as

$$
I_{\mathrm{th}}=\frac{P_{\mathrm{th}}}{E_{980}} \times q \times \frac{1}{f} \sim 0.99 \mathrm{~mA}
$$

where $E_{980}$ is the photon energy at 980-nm wavelength, $q$ is the electron charge, and $f$ is the duty cycle of the pumping beam. The measured $e^{-2}$ transverse mode size from the vertical emission through is about $6 \mu \mathrm{m}$. The estimated $I_{\text {th }}$ of $0.99 \mathrm{~mA}$ is similar as that of long-wavelength VCSELs with a similar mode size. Since the transverse mode is gain-guided in the current hVCL sample, the threshold will be able to be further reduced by introducing a guiding scheme, e.g., a heterostructure in the HCG [3].

Fig. 2(b) shows the in-plane emission profile measured by scanning the single mode lensed fiber along the cleaved plane of the SOI as indicated two white arrows. Considering the Si waveguide is $\sim 1.2 \mathrm{~mm}$ long and the spacing between the end facet of the waveguide and the lensed fiber is $\sim 40 \mu \mathrm{m}$, the profile shows that the emitted light is centered around the in-plane waveguide.

The in-plane emission spectrum at $\sim 4 \mathrm{P}_{t h}$ pumping power is shown in Fig. 3 while the vertical emission spectrum around threshold is shown in the in-set. Higher order transverse modes are strongly suppressed well above the threshold with a side-mode suppression ratio (SMSR) of $27.5 \mathrm{~dB}$. The vertical emission image shows that the lasing mode has a Gaussian-like transverse profile.

\section{Summary}

We have experimentally corroborated the hybrid VCL with in-plane output to a Si waveguide, using CMOS-compatible processes. The laser has similar threshold and single mode properties as single-mode long-wavelength VCSELs. 


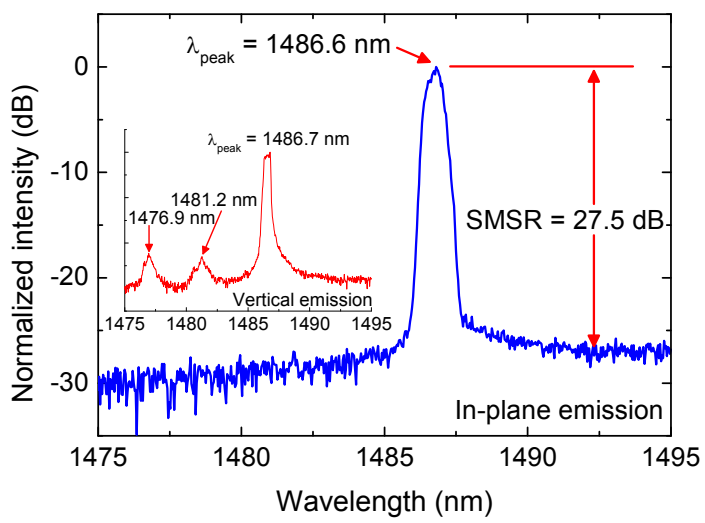

Fig. 3. The measured in-plane output spectra of the hVCL. The inset shows the output spectra measured from the vertical emission.

\section{Acknowledgements}

This work has been supported by the Danish Research Council (Grant No. 11-106620) as well as by the Villum Fonden though the NATEC center.

\section{References}

1. W. H. Hofmann et al., "Energy-Efficient VCSELs for Interconnects," IEEE Photon. J. 4, 652-656 (2012).

2. I.-S. Chung et al., "Silicon-photonics light source realized by III-V/Si-grating-mirror laser," Appl. Phys. Lett. 97, 151113 (2010).

3. C. Sciancalepore et al., "Quasi-3D Light Confinement in Double Photonic Crystal Reflectors VCSELs for CMOS-Compatible Integration,” J. Lightwave Technol. 29, 2015-2024 (2011). 